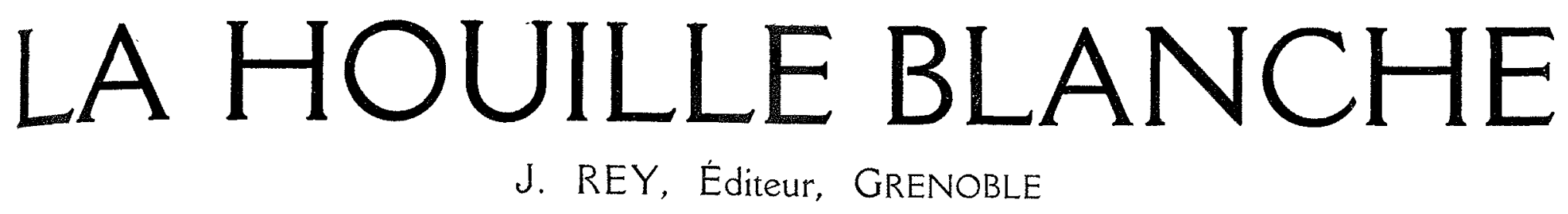

Abonnement pour une Année $\left\{\begin{array}{lll}\text { France... } 30 \text { francs } \\ \text { Etranger. } 40 \text { francs }\end{array}\right\}$ Le Numéro: 5 francs

\title{
SOMMAIRE
}

CONGRES DE MARSEILLE. - I I ligne à 135.000 volts Viccourl-Colmar des Forces Motrices du Haut-Rhin à Mulhouse, par LABBÉ, Ingénieur. - Méthode thermométrique de mesure de rendement des turbines hydrauliques, par F. Porrson, Ingénjeur-Conseil. - Sur l'utilisation des très petiles sources d'énergic hydraulique pour les besoins de l'agriculture, par L. Rigotapd, Ingínicur-agronome. - Les conducteurs électriques d'aluminium el d'aluminium-acier, par M. Dusaugrer, Ingénieur civil des Mines.

CONGRES DE LAMÉNAGEMENT HYDRAULIQUE DU sUD-OUEST. - L'Electrification partielle du réseau de la Compagnie des Chemins cle fer d'Orléans, par M. H. PArodi, Ingénieur al chef des services électriques de la Compagnie d'Orléans.

ÉLECTRICITÉ. - Des diverses applications du régulateur automatique à action rapide Brown-Boveri et des méthodes de branchement correspondantes (suite et fin), par V. Sruvastre, Ingénieur A. M. et I. E. C. - La Traction électrique par accumu-, latenrs, par M. P. Bernabé, Iñgénieur A. et M.
HYDRAULIQUE. - Les lois physiques de l'ćcoulement des fluides, par A. Focr, cliargé du Cours de Physique industrielle à la Faculté des Sciences de Bordeaux. - Débit des déversoirs à contraction latérale (expériences de M. Hégly), par H. B.

LÉGISLATION. - La céssion des concessions de distribution d'énergie. Commentaire de l'article 33 du cahjer lype, par Paul Bougault, Avocat à la Cour cl'Appel de Lyon.

DOCUMENTATION. - Les grands aménagements hydrauliques transalpins, par J. Bouder, Ingénieur A. et M. - L'Electrification des chemins de fer en Italie. - Rôle des réservoirs clans les aménagements hydrauliques. - L'aménagement de la haute vallée du Bergell et du lac des Sils (Suisse). - Un nouveau système de joint assurant l'étanchéité des conduites en béton armé.- Utilisation du four électrique pour la production du fer "anti-rouille". - Anomalies dans les mesures failes aux lubes de Venturi. -

\section{CONGRÉS DE LA HOUILLE BLANCHE et des Applications de l'Électricité}

\section{La Ligne à 135.000 volts Miecourt-Colmar des Forces Motrices du Haut Rhin à Mulhouse.}

Par LABBÉ, Ingénieur à la Sociélé de Force el Lumière électriques, Strasbourg.

But de La Ligne Et GÉnÉralités

Cette ligne a pour but d'importer de l'énergie électrique de Surse en France.

Son point de départ est à Miécourt, en Suisse.

Elle est raccordée au réseau des "Berner Kraftwerke " qui possèdent la station de Olten-Gosgen (Mühlberg) (voir Houille Blanche de janvier-février 1922, nos 61-62). La puissance transportée atteindra $28.000 \mathrm{KW}$ ). Elle alimentera la région de Mulhouse (poste de transformation de l'île Napoléon), les régions de Colmar et Munster, et sera raccordée à Wintzenheim-Turckheim, à côté de Colmar, à la ligne existante à 70.000 volts de la Société d'Electricité de Strasbourg, qui dessert à son tour la région de Sélestat, la région de Strasbourg,celles de Haguenau, Pechelbronn (mines de pétrole), et celle de Schirmeck.

Elle se prolongera ensuite sur Sarrebourg-Creutzwald (mines de la Houve) par une autre ligne à 70.000 volts appartenant à la Salec (Société Alsacienne et Lorraine d'Électricité), actuellement en construction.
De cette façon la centrale à vapeur de la Jouve (inslallée sur le carreau de la mine) recevra des appoints d'énergie de Suisse. Cettc solution scra alors la plus grande interconnection de réseaux existant en France.

Les postes de transformations nécessaires ne sont pas cncore construits, mais le seront prochainement. Actuellement les lignes fỏnctionneront à 70.000 volts (Poste de l'lle Napoléon, Poste de la Holzmatt à Strasbourg, Poste de Haguenau).

A l'lle Napoléon, la ligne à 135.000 volts fonctionnera en parallèle avec celle de Laufenbourg à 70.000 volts - et celle de Rheinfelden à 40.000 volts.

Dans un avenir rapproché nous verrons surgir : la station de Kembs sur le Rhin, et vraisemblablement la station de pompage des lacs blanc et noir dans la vallée de Kaysersberg (Haut-Rhin). A ce sujet il est heureux de constater que la commission du Rhin a enfin accepté la solution française de captation des Forccs du Rhin, malgré les oppositions suisse et allemande.

Pour plus de renseignements, voir la conférence faite par $M$. Kœchlin, ingénieur, à l'Association régionale des Ingénieurs d'Alsace-Lorraine (A. R. I. A. L.). 
Tracé de la Ligne

Cette ligne est divisée en trois portions :

10 Miécourt-Frontière suisse ;

$2^{o}$ Frontière suisse-Ile Napoléon (Mulhouse);

30 Ile Napoléon-Wintzenheim (Colmar).

Le plan ci-joint montre Je tracé de la ligne.

Dans l'étude qui suit, il ne sera parlé que de la portion Ile Napoléon-Wintzenheim (Colmar) actuellement en cours de construction par la Société anonyme de Force et Lumière électriques de Strasbourg.

La ligne aboutit à la sous-station de l'Ile Napoléon à un pylonè d'angle, dont il sera parlé plus loin, croise la route nationale de Strasbourg à Bâle et oblique vers le Nord-Ouest pour croiser à nouveau cette même route au Nord d'Ensisheim, ce qui représente un alignement droit, de près de 20 kilomètres; ensuite jorme un coude vers l'Ouest de Sainte-Croix-en-Plaine pour éviter la forèt de la Thur et arrive à Wintzenheim par-deux aligneuents droits de 10 kilomètres.

La portée moyenne est de 210 mètres.

Pylones. - Les dimensions des pylones sont indiquées dans le tableau ci-joint:

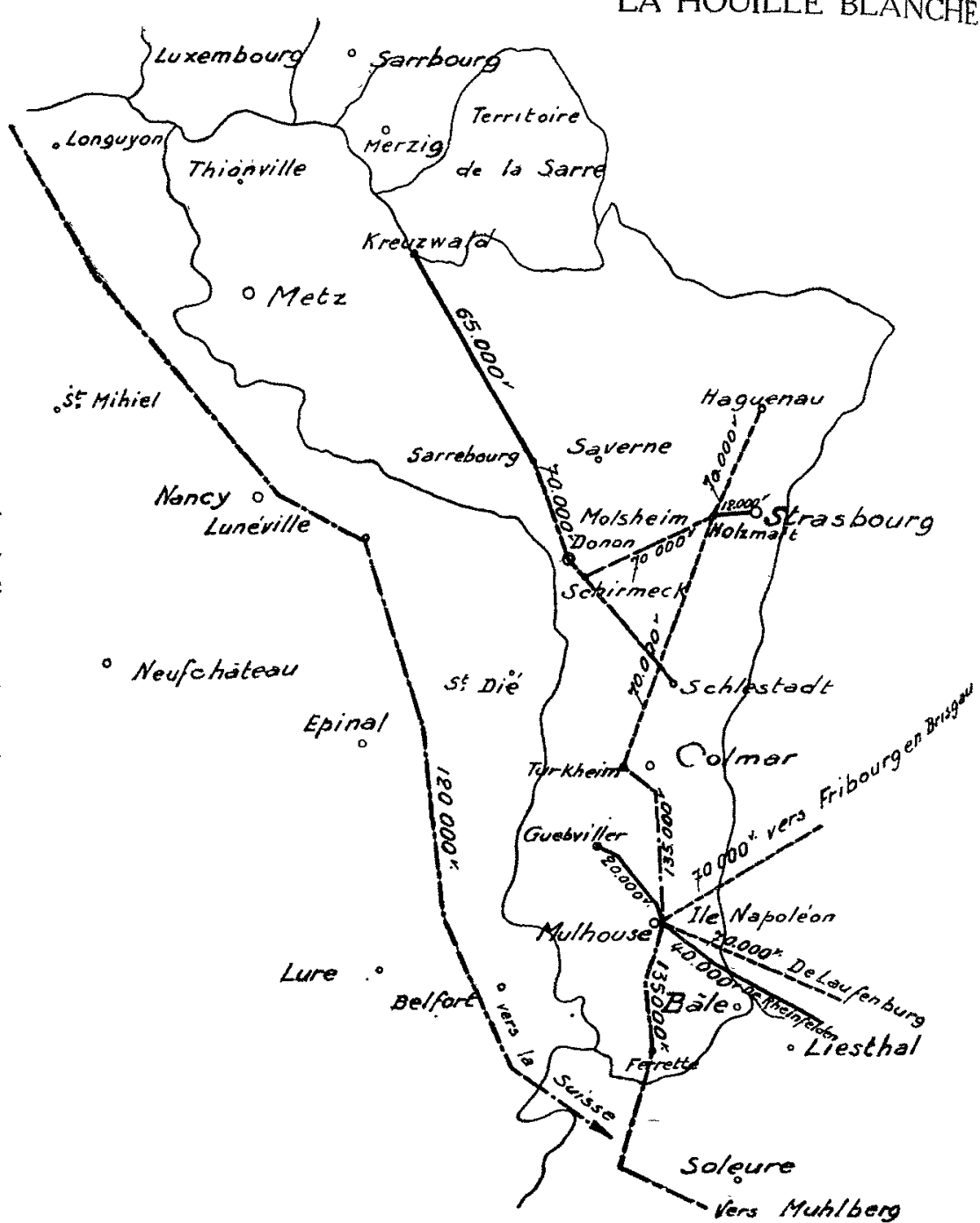

Tableau comparatif des caractéristiques des pylônes de la ligne d'état à 120.000 volts et de la ligne 135.000 volts Miecourt-Colmar.

\begin{tabular}{|c|c|c|c|c|c|}
\hline \multirow[b]{2}{*}{ "DESTGNATION } & \multicolumn{2}{|c|}{120.000 volts (socle béton) } & \multicolumn{3}{|c|}{135.000 volts (implanlalion en terre vierge) } \\
\hline & $\begin{array}{c}\text { Pylône } \\
\text { d'alignement }\end{array}$ & $\begin{array}{c}\text { Pylône } \\
\text { d'amarrage ou } \\
\text { d'angle }\end{array}$ & $\begin{array}{c}\text { Pylône } \\
\text { d'alignement }\end{array}$ & $\begin{array}{c}\text { Pylône renlorcé } \\
\text { pour } \\
\text { traversée de route }\end{array}$ & Pylône d'angle \\
\hline Dimensions des bases . & $1,55 \times 1,55 \mathrm{~m}$ & $2,20 \times 2,20 \mathrm{~m}$. & $1,807 \times 1,807 \mathrm{~m}$. & $1,900 \times 1,900 \mathrm{~m}$. & $4,60 \times 4,60$ \\
\hline Dimensions des têtes $\ldots \ldots \ldots \ldots$ & $0,50 \times 0,50 \mathrm{~m}$ & $0,60 \times 0,60 \mathrm{~m}$ & $0,70 \times 0,70$ & $0,70 \times 0,70$ & 4 pieds \\
\hline Tronçon inférieur. Poirls $\ldots \ldots \ldots \ldots$ & $819 \mathrm{kgs}$ & $1,517 \mathrm{kgs}$ & $2340 \mathrm{kgs}$ & $2.894 \mathrm{kgs}$ & - \\
\hline Longueur $\ldots \ldots \ldots \ldots \ldots \ldots \ldots$ & $7,50 \mathrm{~m}$ & $6,83 \mathrm{~m}$ & $7,765 \mathrm{~m}$ & $7,765 \mathrm{~m}$ & - \\
\hline Cornières des montants .......... & $90 \times 90 \times 9 \mathrm{~m} / \mathrm{m}$ & $110 \times 110 \times 13^{\mathrm{m} / \mathrm{m}}$ & $90 \times 90 \times 9$ & $100 \times 100 \times 10$ & $120 \times 120 \times 12$ \\
\hline Cornières des croisillons ........... & $60 \times 60 \times 6 \mathrm{~m} / \mathrm{m}$ & $80 \times 80 \times 8 \mathrm{~m} / \mathrm{m}$ & $60 \times 60 \times 6$ & $60 \times 60 \times 6$ & - \\
\hline Rivets $\ldots \ldots \ldots \ldots \ldots \ldots \ldots \ldots$ & $18 \mathrm{~m} / \mathrm{m}$ & $24 \mathrm{~m} / \mathrm{m}$ & $16 \mathrm{~m} / \mathrm{m}$ & $18 \mathrm{~m} / \mathrm{m}$ & - \\
\hline Tronçon milieu. Poids . . . . . . . . . . & $512 \mathrm{kgs}$ & $893 \mathrm{kgs}$ & $750 \mathrm{kgs}$ & $930 \mathrm{kgs}$ & - \\
\hline Longueur $\ldots \ldots \ldots \ldots \ldots \ldots \ldots$ & $7,185 \mathrm{~m}$ & $6,97 \mathrm{~m}$ & $7,755 \mathrm{~m}$ & $7,755 \mathrm{~m}$ & Hauleur totale \\
\hline Cornieres des montants & $80 \times 80 \times 8 \mathrm{~m} / \mathrm{m}$ & $100 \times 100 \times 12^{\mathrm{m} / \mathrm{m}}$ & $80 \times 80 \times 8$ & $90 \times 90 \times 9$ & $26 \mathrm{~m}$ \\
\hline Cornieres des croisillons & $50 \times 50 \times 5 \mathrm{~m} / \mathrm{m}]$ & $70 \times 70 \times 7 \mathrm{~m} / \mathrm{m}$ & $60 \times 60 \times 6$ & $60 \times 60 \times 6$ & - \\
\hline Rivets $\ldots \ldots \ldots \ldots \ldots \ldots$ & $16 \mathrm{~m} / \mathrm{m}$ & $22 \mathrm{~m} / \mathrm{m}$ & $16 \mathrm{~m} / \mathrm{m}$ & $18 \mathrm{~m} / \mathrm{m}$ & 一 \\
\hline Tronçon supéricur. Poids . ........ & $376 \mathrm{kgs}$ & $806 \mathrm{kgs}$ & $1.130 \mathrm{kgs}$ & $1390 \mathrm{kgs}$ & - \\
\hline Longueur $\ldots \ldots \ldots \ldots \ldots \ldots$ & $8,58 \mathrm{~m}$ & $9,44 \mathrm{~m}$ & $12,060 \mathrm{~m}$ & $12,060 \mathrm{~m}$. & - \\
\hline Cornières des montants ... & $70 \times 70 \times 7 \mathrm{~m} / \mathrm{m}$ & $90 \times 90 \times 10^{\mathrm{m} / \mathrm{m}}$ & $\begin{array}{l}70 \times 70 \text { et } \\
60 \times 60 \times 6\end{array}$ & $1399 \mathrm{kgs}$ & - \\
\hline Cornières des croisillons & $40 \times 40 \times 4 \mathrm{~m} / \mathrm{m}$ & $60 \times 60 \times 6 \mathrm{~m} / \mathrm{m}$ & $50 \times 50 \times 5$ & $50 \times 50 \times 5$ & - \\
\hline Rivets .................. & $14 \mathrm{~m} / \mathrm{m}$ & $20 \mathrm{~m} / \mathrm{m}$ & $16 \mathrm{~m} / \mathrm{m}$ & $16 \mathrm{~m} / \mathrm{m}$ & - \\
\hline Poids des bases $\ldots \ldots \ldots \ldots \ldots \ldots \ldots$ & $400 \mathrm{kgs}$ & $800 \mathrm{kgs}$ & - & - & - \\
\hline $\begin{array}{l}\text { Nombre de boulons de } 3 \mathrm{~m} / \mathrm{m} \text { de fixation } \\
\text { sur le socle } \ldots \ldots \ldots \ldots \ldots \ldots \ldots\end{array}$ & 24 & 40 & Traverses Zores 8 & $\mathrm{~d}^{0}$ & Béton $50 \mathrm{~m}$. \\
\hline & $485 \mathrm{kgs}: \mathrm{m} / \mathrm{m} 2$ & $4 \mathrm{kgs}: \mathrm{m} / \mathrm{m} 2$ & $\begin{array}{c}\text { à } 10 \text { Comp. } 4.200 \mathrm{k} . \\
\text { envïron }\end{array}$ & $\begin{array}{c}\text { Complet } 5.200 \mathrm{~kg} \\
\text { environ }\end{array}$ & Complet $7.650 \mathrm{~kg}$ \\
\hline Poids du pylône hors sol . . . . . . . . . . & $2.118 \mathrm{kgs}$ & $3.938 \mathrm{kgs}$ & & & \\
\hline $\begin{array}{l}\text { Effort maximum du sommet sur les } \\
\text { pylônes } \ldots \ldots \ldots \ldots \ldots \ldots \ldots \ldots \ldots\end{array}$ & $2.235 \mathrm{kgs}$ & 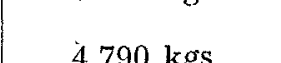 & $2350 \mathrm{kgs}$ & $1800 \mathrm{kgs}$ & - \\
\hline Angle de la ligne possible ........... & 0 degré & 160 degrés & & & \\
\hline $\begin{array}{l}\text { Travail maximum du métal } \ldots \ldots \ldots \ldots \\
\quad \text { dans les montants } \ldots \ldots \ldots \ldots \ldots\end{array}$ & $12 \mathrm{kgs} \mathrm{m} / \mathrm{m}$ & $12 \mathrm{kgs} \mathrm{m} / \mathrm{m} 2$ & $\begin{array}{l}13,36 \mathrm{~kg} \mathrm{~m}^{\mathrm{m}} / \mathrm{m}^{2} \\
\text { en CG' (voir doss } \mathrm{n} \text { ) }\end{array}$ & $\begin{array}{l}\text { Coefficient de sé- } \\
\text { curité })>5\end{array}$ & - \\
\hline
\end{tabular}


De semblables pylones ont été décrits dans le R. G. E. du II novembre 1921 , tome $X, n^{0} 20$, pour les lignes à 120.000 volts. C.eux employés sur la ligne Ile Napoléon-Wintzenheim sont plus forts ct plus grands encore que ceux décrits.

La particularité de ces appuis consiste dans l'absence de béton, pour en assurer la stabilité.

Lc pied est en une seule pièce (et non articulé), il est muni de traverses de chemin de fer en acier. Les pylones d'angle et d'amarrage sont maintenus par 4 socles de béton de 13 mètres cubes chacun, adaptés aux 4 pieds.

La profondeur des trous est de $2 \mathrm{~m} .25$.
Sur la portion Colmar-Ile Napoléon, l'économic réalisée est supéricure à $1.000 \mathrm{fr}$. par pylone (Voir le calcul justificatir à la fin du présent rapport).

Il faut surveiller avec beaucoup de soins l'application de l'asphalte, et veiller à ce que toutes les parties soient bien recouvertes d'une couche d'au moins $4 \mathrm{~m} / \mathrm{m}$ d'asphalte. Cette couche plus ou moins régulière atteint $8 \mathrm{~m} / \mathrm{m}$ à certains endroits. Nous indiquons à la fín de ce rapport la meilleure façon d'appliquer l'asphalte.

Les têtes et bras sont galvanisés par immersion à chaud d'une couche épaisse de galvanisation, devant résister à 5 pliages sans s'écailler.

A ce sujet, nous indiquons à la fin du présent rapport le cahicr

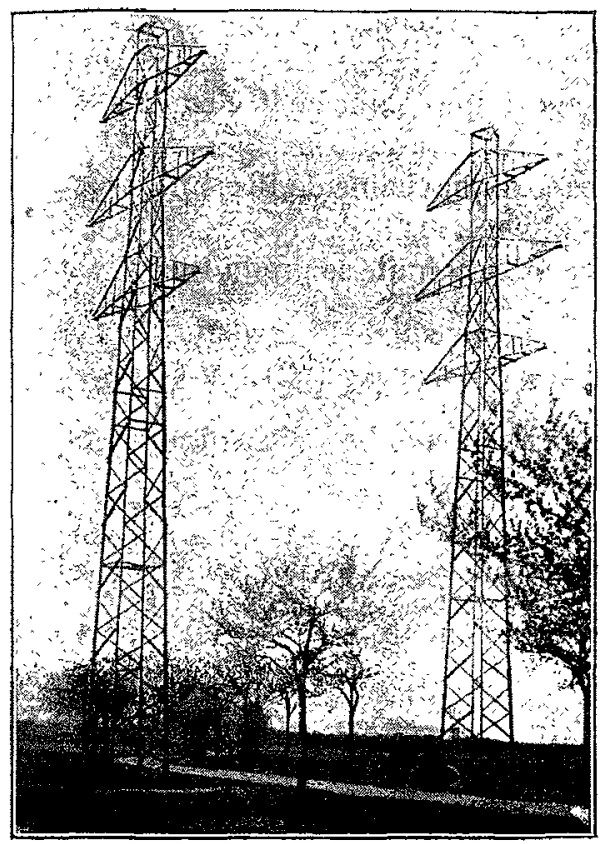

Vue des pylônes de traversée de route départementale.

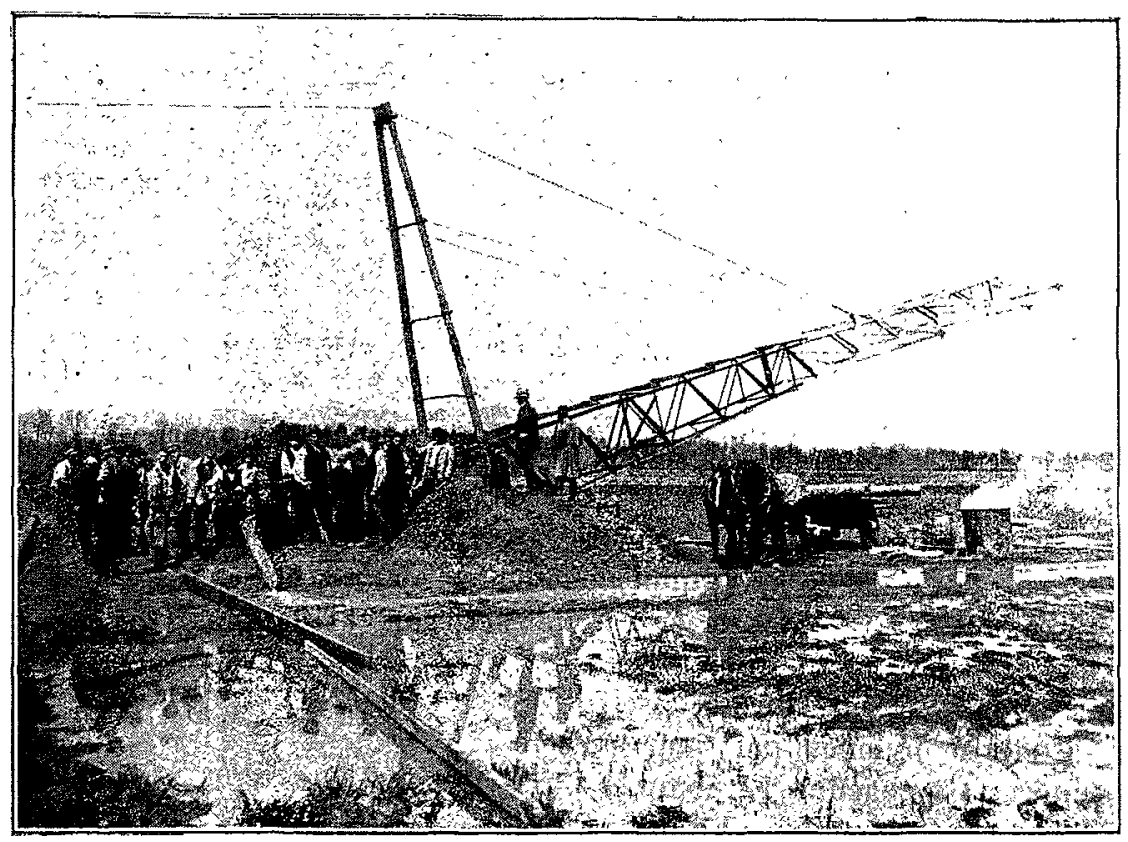

Montage d'un pylône.
Avant d'enterrer les pieds, les fers reçoivent deux couches d'asphalte spécial.

Il existe deux produits : L'un des produits est utilisé à chaud, l'autre à froid, quand la température le permet. Le produit à chaud s'applique beaucoup mieux, sèche rapidement (dix minutes), reste plastique, adhère mal si les parties métalliques contre lesquelles on l'applique ne sont pas bien sèches ; c'est pourquoi il a été prévu un goudron spécial à froid pour les applications par temps de pluies.

Cette façon de faire avait été employée déjà pour les pylones de la ligne "Laufenbourg-Ile Napoléon ", et avait donné de bons résultats. Des pylones implantés depuis plus de 10 ans étaient parfaitement conservés, au moment où la décision fut prise pour choisir entre le béton et l'asphalte pour la ligne 135.000 volts.

Il est plus facile d'employer l'asphalte que le béton ; il exige moins d'hommes, mais l'application en reste néanmoins très coûteuse, varie de 6 à $10 \mathrm{fr}$. le m2. Son emploi est plus économique que celui du béton. L'économie est d'autant plus sensible que les difficultés de transport à pied d'œuvre sont plus grandes. Les matériaux constituant le béton coûtent très cher de transport. Nous avons constaté des différences de prix atteignant $70 \%$ dans le prix du béton pour les lignes accidentées.

En revanche, l'emploi de l'asphalte exige un pied de pylone renforcé et lourd, qu'on peut économiser, si on emploie le béton.

De plus, les pylones étant très lourds, une étude géologique des terrains est nécessaire pour reconnaître la résistance du sol qui ne doit pas être mouvant et offrir une résistance d'au moins $1 \mathrm{~kg} 5$ par cmq. des charges concernant la galvanisation des pylones, aiısi que quelques renseignements sur la galvanisation en général.

\section{Construction de la Ligne}

Les pylones, vu leur encombrement, ont été livrés en tronçons de faible longueur et en parties dètachées.

La mise à pied d'œuvre s'est faite au moyen de voitures de paysans ; l'assemblage des pylones a eu lieu sur place.

La mise en direction pour le levage a été faite à l'aide de simples crics de 4 tonnes.

Le levage a eu lieu au moyen de treuils mobiles, dont le câble de traction fixé aux bras médians passe au sommet d'une chèvre articulée aux pieds sur un axe fixe, et fixée au pylone lui-même par 4 tendeurs.

Le pylone repose sur un axe, par deux coussinets spéciaux, fixés par brides aux montants principaux du pylone. La forme des coussinets a été étudiée sur place, lors du dressage du premier pylone (Procédé de Sté An. de Force et Lumière Electr.) Une fois une rotation de 0 à $45^{\circ}$ du pylone autour de cet axe obtenue, le câble de traction quitte la tête de la chèvre mobile, le pylone continue à tourner autour des coussinets, jusqu'à ce que les traverses viennent heurter l'angle du fond du trou, la rotation continue par pivotement autour des extrémilés des traverses. Le coussinet et la chèvre mobile quittent alors l'axe employé pour la première rotation de 0 à $45^{\circ}$.

La durée de levage proprement dite varie de 10 à 25 minutes, suivant la nature du terrain et le temps. En moyenne il faut 
compter 15 minutes. Certains entrepreneurs ont fait micux, mais ce qu'il ne faut pas oublier de dire, c'est qu'il ne s'agit pas de gagner des minutes sur le levage, - mais des minutes, voire même des quarts d'heure, sur le transport du matériel de pylone à pylone, sur l'installation de l'appareil de dressage, sur la mise en direction, sur l'alignement, etc... Il faut, en tenant compte

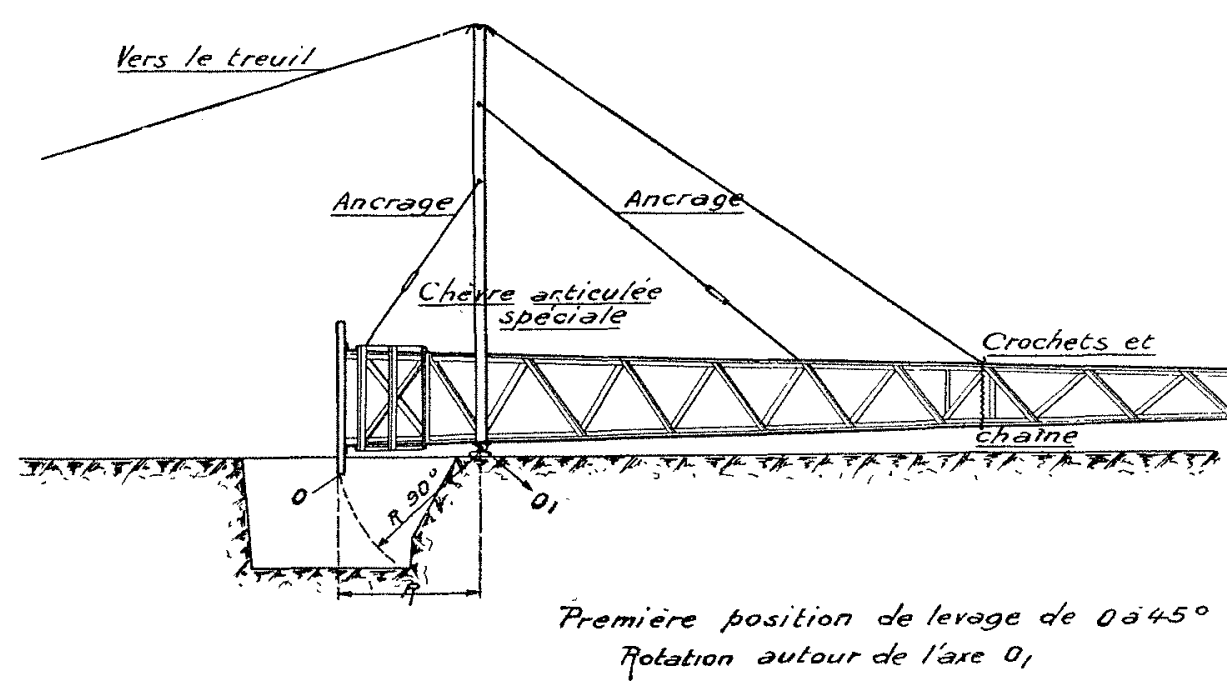

Dispositif "Forclum " pour le dressage des grands pylônes.

\section{ISOLATEURS}

Jes isolateurs du type à chaine et Ohio Brass type 250 nioffrent rien de particulier sur ceux connus et emplovés jusqu'ici. Leurs caractéristiques sont les suivants :

Nombre d'éléments : 5 et 6 suivant le pylone.

\section{$1^{\circ}$ Conditions électriques :}

Amorçage de l'arc sur l'élément à sec Amorçage de l'arc sur l'élément sous pluie..$\ldots \ldots \ldots \ldots \ldots \ldots$

Amorçage d'une chaine de 5 éléments

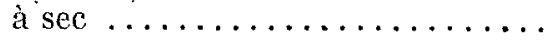

Amorçage d'une chaine de 5 éléments sous pluie .............

Amorçage d'une chaine de 10 éléments à $\sec \ldots \ldots \ldots \ldots \ldots \ldots \ldots$ Amorçage d'une chaine de 10 éléments sous pluie .............. $325000 \mathrm{v}$ Percement sous huile se produit vers. $140000 \mathrm{v}$.

(Voir à ce sujet le Bulletin de l'Association suisse des Electriciens 1921, no 11).

Un cerlain nombre d'isolaleurs " Hormsdorf ont élé employès.

$2^{\circ}$ Résistance mécanique :

do tout ceci, compler sur 3 à 4 heures, par pylone suivant le terrain el le temps. C'est pourquoi nous nous sommes efforcé de rendre l'outillage le plus léger possible, et partant le plus transportable possible.

Nous avons atteint une vitesse dc dressage de 5 pylones par jour avec deux équipes de chacune 12 hommes les pylones étant assemblés. La ligne de 42 kilomètres a eu tous les pylones dressés en 40 jours effectifs. Le tirage des câbles aura demandé également 60 jours effectifs.

Les pylones d'angles ont été dressés d'une scule pièce, sans les bras inférieurs et supérieurs, - ce qui, notons-le, représente une tour Eiffel de 7.700 kilogs et de $28 \mathrm{~m}$. de hauteur. Etant donné la disposition des pieds, des fers de renforcement avaient été fixés entre les pieds situés dans les plans parallèles au plan du câble de traction. La durée de dressage d'un tel pylone a demandé unc heure, car pour arriver à développer l'effort nécessaire qui est de l'ordre de $10 \mathrm{~T}$., nous avons eu recours à des engins de démultiplication, puisque nous avons déjà fait remarquer que nous nous étions efforcés d'alléger le plus possible l'ensemble dę l'outillage.

Il est curieux de noter en passant qu'il faut deux charpentes pareilles, situées à 15 mètres l'une de l'autre environ, pour traverser les petites routes et les yoies ferrées secondaires à une voie, ce qui représente une dépense énorme; de plus, il faut éviter les angles qui sont très onéreux. Un pylone d'angle revient à environ 13.000 francs nu et implanté (monlage seulement).

L'alignement et l'exécution ont été tels qu'une erreur de $60 \mathrm{c} / \mathrm{m}$ a été constatée sur le dernier pylone ; cette erreur est négligeable, si on tient compte de la longueur de $20.000 \mathrm{~m}$. de l'alignoment. Elle a été facilement compensée par le pylone d'angle.
Charge à la rupture de l'élément ............ $4100 \mathrm{~kg}$

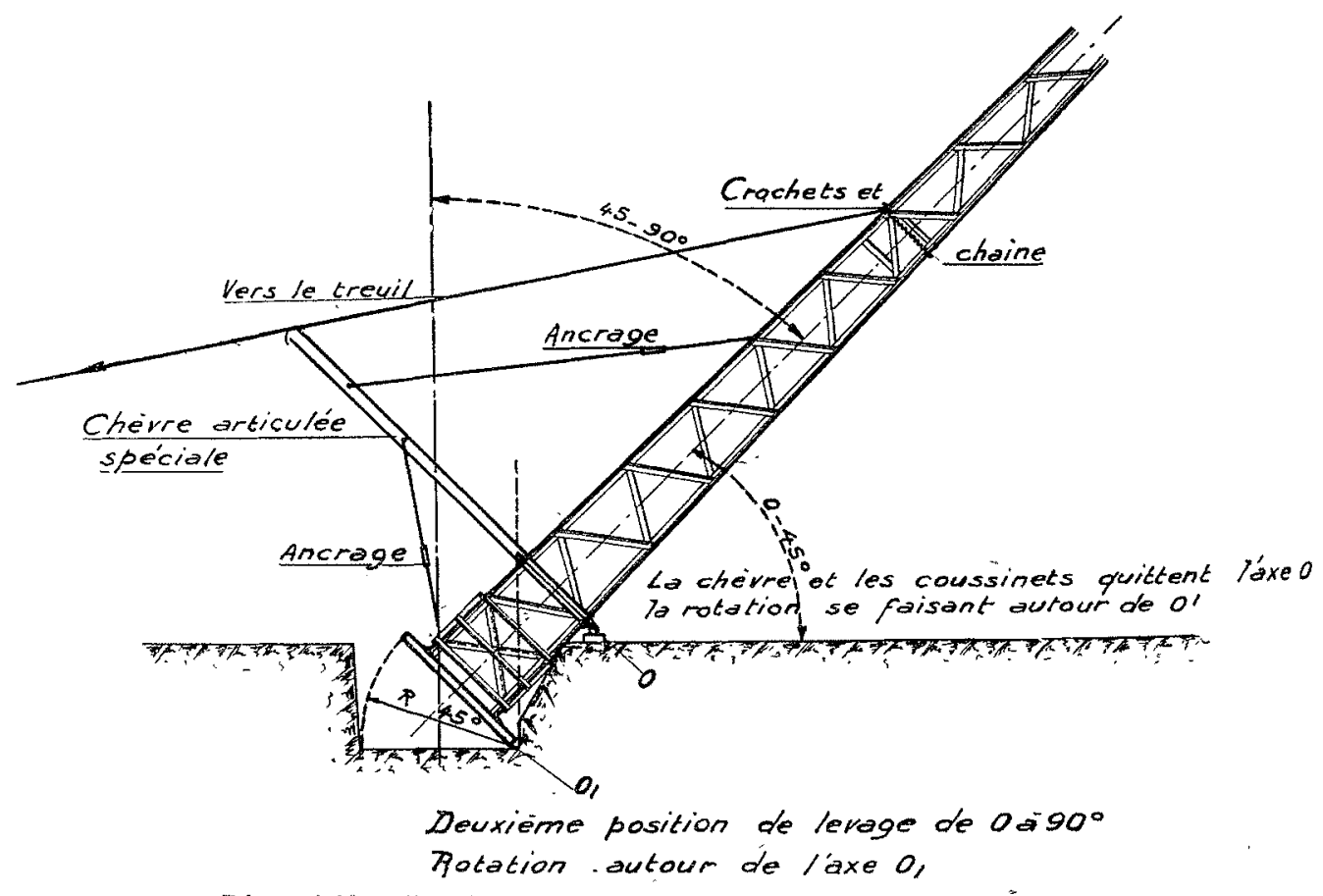

Dispositif" "Forclum "pour le dressage des grands pytônes.

\section{Conducteurs}

Il est employé trois câbles aluminium acier de $210 \mathrm{~m} / \mathrm{m}^{2}$ de section. $\Pi$ en est prévu six.

L'accrochage aux isolateurs est obtenu au moyen de pinces spéciales, recouvrant le câble sur environ $30 \mathrm{~cm}$. Celui-ci est garni d'une fourrure en tôle d'aluminium pour diminuer la pression sur le câble lui-même et pour augmenter l'adhérence. Les manchons de jonction sont du type Payrard avec coins de coincement.

Les précautions à prendre et dont il a déjà été rendu compte dans la $R . G . E$. du 19 novembre 1921 , tome $\mathrm{X}, n^{\circ} 20$, ansi que 
dans les brochures de l'Aluminium Français par M. Dusaugey sont à observer rigoureusement. A cet effet toute une éducation spéciale des ouvriers est à faire.

\section{Les caractéristiques sont les suivants :}

Câble aluminitum-acier . . . . . . . . . . . . 210,3 m/m²

Section câble acier.............. $39,8 \quad$ -

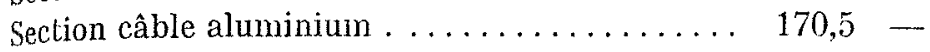

Nombre des brins câble acier . . . . . . . . . 7

Nombre des brins câble aluminium . . . . . . . 30

Longucur de fabrication ............. $1400 \mathrm{~m}$

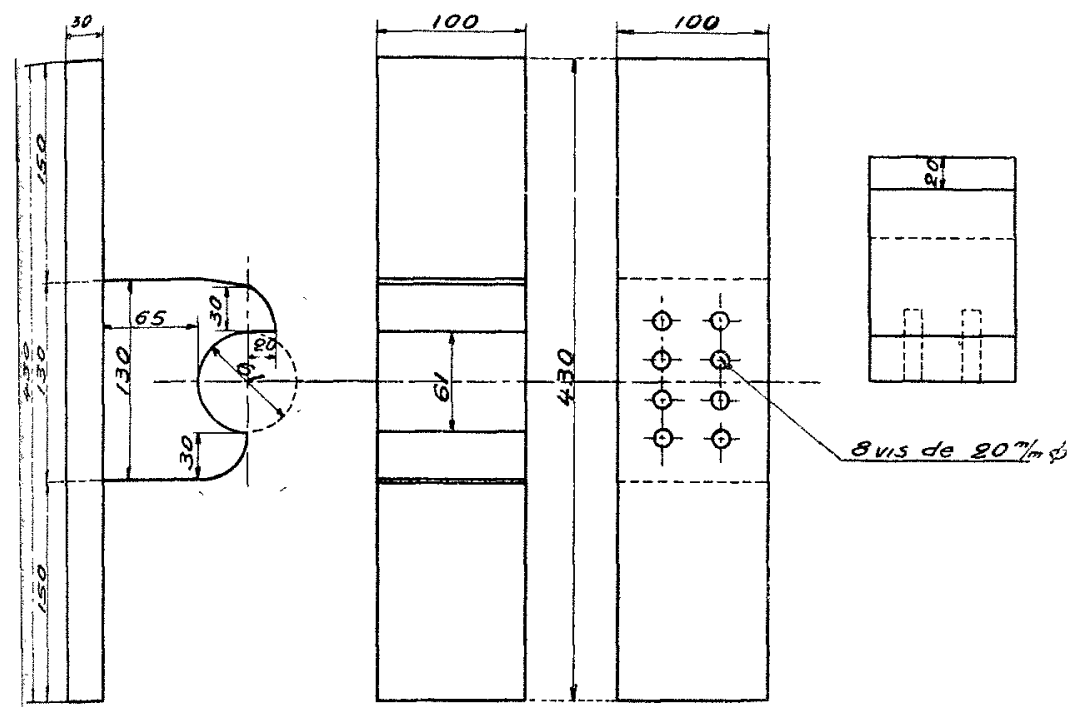

Coussinet spécial "Forclum " pour le dressage de pylônes lourds.

\section{$1^{\circ}$ Propriétés chimiques:}

Aluminium type dit à 99,3\% d'aluminium pur. Somme des teneurs en fer et silicium, pas supérieure à $0,7 \%$, sauf écarts tolérés de $0,3 \%$.

$2^{\circ}$ Propriétés mécaniques :

Résistance à la rupture, fils acier ......... $120 \mathrm{kgs} / \mathrm{mm}^{2}$

Résistance à la rupture, fils aluminium-écroni . $20-$

Charge à la rụpture du câble acier ......... 4746-kgs

Charge à la rupture du câble aluminium ... . . 3410 .

Charge à la rupture du câble mixte . . . . . . . 7000 _.

Module de Young fils acier ............ $22.10^{5} \mathrm{k} / \mathrm{cm}$

Module de Young fils aluminium . . . . . . . 7,15.10

Limite d'élasticité fil acier . . . . . . . . . $9500 \mathrm{kgs}$

Limite d'élasticité fil aluminium .......... 1100 --

Coefficient de délation linéaire fils acier $\ldots \ldots \ldots \quad 11,5.10^{-6}$

Coefficient de délation linéaire fils aluminium .. $22,8 \times 10^{-6}$

Coefficient de délation linéaire câble mixte. . . 17,25 $\times 10^{-0}$

Allongement à la rupture fils acier ......... $>6 \%$

Allongement à la rupture fils aluminium...... $>2 \%$

Mesuré sur éprouvette de $20 \mathrm{~cm}$. de longueur.

\section{Propriétés électriques :}

Conductibilité fil aluminium à $15^{\circ} \mathrm{C}$

Résistance spécifique pour un fil al. $15^{\circ}$

Coefficient de température de la résisdivité.....

Cable acter $70 \mathrm{~m} / \mathrm{m}^{2}$

Section câble en acier. ..................

Nombre de brins $7+12 \ldots \ldots \ldots \ldots \ldots \ldots \ldots \ldots$

Diamètres des fils ....................

Diamètre extérieur du câble . . . . . . . . . . .

Longueur de fabrication
10 Propriétés mécaniques :

Résistance suplure fills acier............ $140 \mathrm{kgs} / \mathrm{m} / \mathrm{m}^{\mathrm{w}}$

Résistance suplure câble............. 120 -

Charge à la suplure câble ... . . . . . . . 8400 kgs

Module d'électricité fil acier. . . . . . . . . . $22-10^{5} \mathrm{~kg} / \mathrm{cmq}$

Limite d'électricité fil acier . . . . . . . . . . . . . 11000 -

Coeff. dilatation fil acier $\ldots \ldots \ldots \ldots \ldots \ldots \ldots \quad 11,5-10^{-6}$

Allongement à suplure des fils $\ldots \ldots \ldots \ldots \ldots \ldots>6 \%$

$2^{\circ}$ Propriétés électriques:

Conductibilité à $20^{\circ}$ C. environ $\ldots \ldots \ldots \ldots \ldots \ldots 5 \frac{m}{\Omega \mathrm{m} / \mathrm{m}=}$

\section{DÉroulemENT}

Le déroulement s'effectue au moyen d'un chariot spécial, câble par câble. Le câble est hissé aux bras sur des roulettes en bois spéciales, et réglées pour être à la même hauteur que les pièces d'attache des isolateurs. Le réglage est fait à l'aide du dynamomètre, du théodolithe et au moyen de nivelettes mobiles, spécialement construites à cet effet.

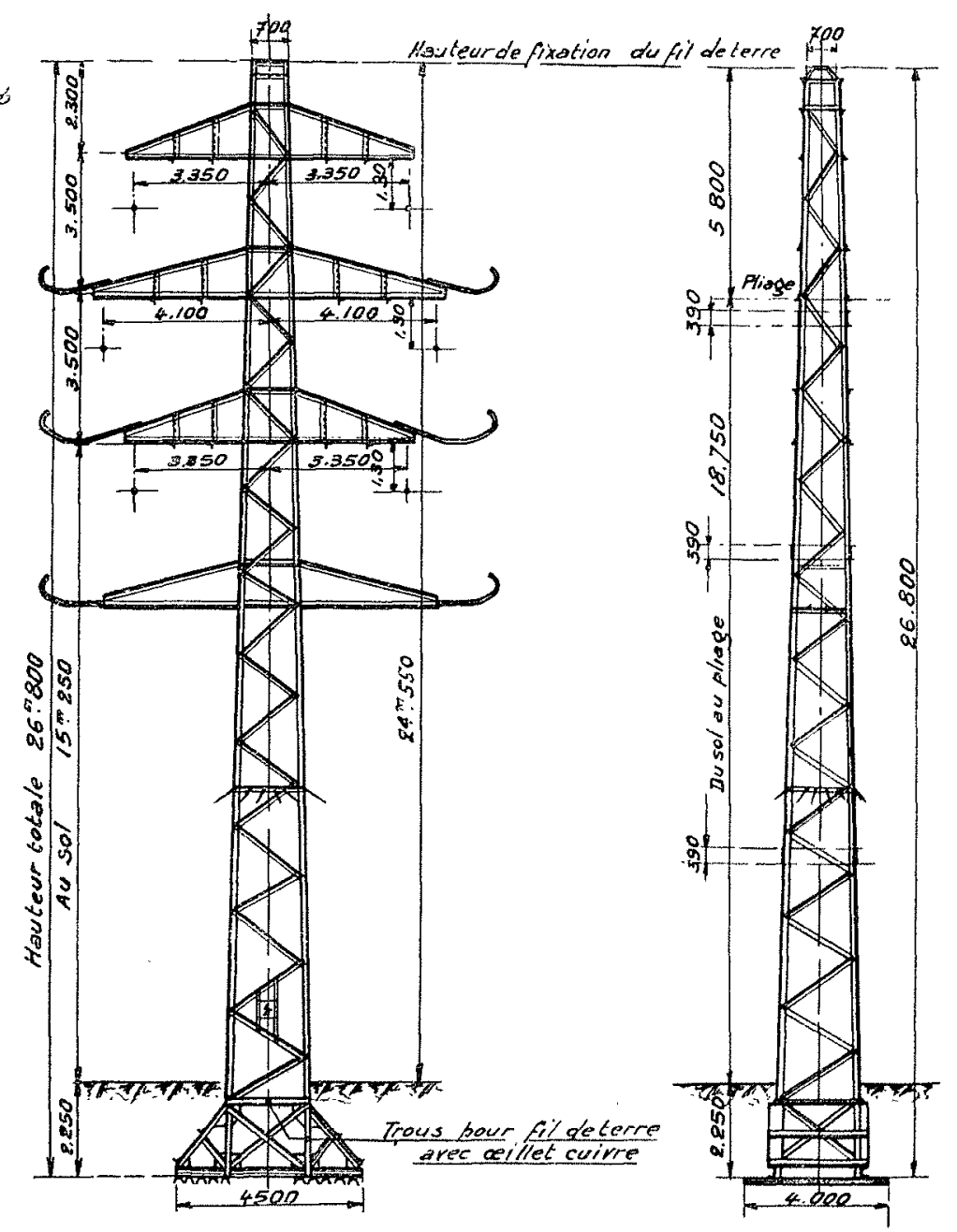

Pylône renforcé.

La flèche maxima de 6 mètres $\mathrm{a}+45^{\circ}$. La tension maximum du câble aluminium-acier est de $9 \mathrm{kgs}$ par $\mathrm{m} / \mathrm{m}^{2}$ dans le cas le plus défavorable.

Le câble de garde est constitué par un câble acier de $70 \mathrm{~m} / \mathrm{m}^{2}$. La fixation n'offre rien de particulier.

\section{Communications TÉLÉPhoniques}

Des essais de communications radiotéléphoniques avec dispositif d'appel à distance sont en cours. Les résultats obtenus jusqu'ici sur d'autres lignes donnent bon espoir. (II s'agit de dispositifs nouveaux). 


\section{ASPHALTAGE}

Nous indiquons ci-après les renseignements relatifs à l'asphallage qui nous ont été gracieusement communiqués par la Société Strasbourgeoise des Asphaltes et Bitumes.

Pour être sûr d'une bonne conservation, il faut employer du bitume et non un dérivé quelconque de la distillation de la houille, quoique ces dérivés soient improprement appelés bitume. Les bitumes sont des asphaltes purs trouvés dans la nature, (qui gardent leurs qualités et propriétés éternellement (Dans les ruines de Babylone et Ninive des murs ont été retrouvés, dont les pierres étaient liées au mastic bitumeux encore aujourd'hui en un état de parfaite conservation. Ils étaient autrefois recueillis à la surface de la mer Morte en Judée).

La meilleure manière d'asphalter les pylones pour obtenir de bons résultats doit se faire de la manière suivante :

Les parties métalliques sont avant tout à nettoyer avec une brosse, afin d'enlever toute partie terreuse. Un premier enduit est à donner avec la malière asphaltique préparée d'une manière spéciale la rendant fluide et apte à être répandue sans l'aide du feu, c'est-à-dire à froid. Ce premier enduit doit sécher au bout de peu de temps et a pour but : $1^{\circ}$ de recouvrir toutes les parties du fer, et $2^{\circ}$ de former une base d'adhérence pour le second enduit.

Celui-ci consiste en un bitume raffiné, tenace et collant, fabriqué spécialement avec du bitume de Trinité. Il est rendu fluide dans des chaudières à feu direct et appliqué en deux couches sur une épaisseur totale de $2-3 \mathrm{~mm}$. Il est sec une heure environ après l'application.

\section{Gialvanisation}

Le Zingage, improprement appelé "Galvanisation ", se fait en général par qualre procédés :

$1^{\circ}$ Le procédé à chaud le plus ancien et le plus simple;

$2^{\circ}$ Le procédé électrolytique, coûteux.

$3^{\circ}$ Le procérlé par cémenlation ou Shérardisation,peu pratique et irrégulier ;

$4^{\circ}$ Le procédé par pulvérisation, ou précédé Shoop, peu adhérent el poreux.

Le Zingage est très employé actuellement pour protéger contre la rouille les parties de pylones difficilement accessibles, quand les lignes sont sous tension; on passe généralement en peinture les parties inférieures, yu'on peut facilement entretenir et surveiller.

Le Zingage n'a pas encore fait ses preuves; personnellement nous sommes convaincu de son succès pour l'avenir et souhaitons que cette pratique se généralise partout.

La galvanisation à chaud est très économique et donne, si elle est bien faite, d'cxccllents résultats. Le zinc ne s'écaille pas sous le choc ni au pliage. Tout le secret de la fabrication réside dans la préparation dı bain (Zinc aussi pur que possible).

Le callier des charges suivaní était imposé pour la galvanisation des pylones de la ligne 135.000 volts :

10 Toute la partie des pylones jusqu'à une hauteur de $3 \mathrm{~m}$. au-dessous du point d'attache des isolateurs sera galvanisée à chaud ; la galvanisation devra être bien adhérente et devra résister à l'épreuve suivante :

La pièce à essayer sera nettoyće au sulfure de carbone,à la benzine ou à l'essence de thérébentine, puis rincée à l'eau claire et cssuyée avec un chiffon de coton ; elle sera ensuite plongée dans une solution de sulfate de cuivre pendant une minute à cinq reprises différentes. Après chaque immersion, la couche noire sera enlevée à l'eau èt à la brosse, puis la pièce sera essuyée aveo un chiffon de coton. La galvanisation sera considérée comme insuffisante si, après la cinquième immersion, brossage et es- suyage, ia pièce présente une couche brillante de cuivre couleur saumon.

On se servira, pour cet essai, de sulfate de cuivre du commerce et la solution comprendra 300 gr. de sulfate par litre d'eau, sa densité après neutralisation par un excès d'oxyde de cuivre et àprès filtrage sera ramenée à 1,186 avant chaque essai,par addition soit d'eau, soit de solution plus concentrée.

Les P. T. T. imposent le cahier des charges suivant :

$2^{\circ}$ Les objets galvanisés devront supporter, sans que le fer soit mis à nu, même partiellement, quatre immersions d'une minute chacune dans une dissolution de sulfate de cuivre dans cing fois son poids d'eau, la température de la dissolution au moment de l'expérience étant de 15 degrés.

Le zinc ne devra pas s'écailler sous le choc.

Toutes les pièces qui ne satisferont pas à ces essais ou porteront des taches de rouille seront rejetées.

Nous avons assisté à des essais de réception chez MM.Stoffler frères à Strasbourg. La pièce galvanisée laissait apparaître la couleur saumon à la onzième immersion.

Tous les constructeurs de pylones ne sont pas organisés pour galvaniser. Le procédé le plus pratique est le procédé Shoop, caz il est portatif et tout le monde peut l'appliquer ; mais nous ne le préconisons pas et avons constaté que l'immersion à chaud Stoeffler était supérieure.

La maison Stoeffler a organisé un service de galvanisation sur place qui est capable de s'installer dans n'importc quelle usine fabriquant des pylones.

Le prix de revient de la galvanisation est d'environ fr. 38 les $100 \mathrm{kgs}$.

Calcul de l'économie réalisée pour un pylone d'alignement en employant l'asphalte :

Coût d'implantation d'un pylone à pied asphalté :

10 Dressage d'un pylone de $5.000 \mathrm{kgs}$ environ .......

$2^{\circ}$ Terrassement nécessaire $50 \mathrm{~m} 3$ à $8 \mathrm{fr} . \ldots \ldots \ldots \ldots$ $600 \mathrm{fr}$.

$3^{\circ}$ Supplément pour le pylone bétonné pour renforcement du pied $\ldots \ldots \ldots \ldots \ldots \ldots \ldots \ldots \ldots$

$4^{0}$ Asphaltage $\ldots \ldots \ldots \ldots \ldots \ldots \ldots \ldots \ldots \ldots$

$5^{\circ}$ Supplément pour transport à pied d'wuvre du supplémént de port ...............

Total ................. 400

1.000

$600 \%$

20

$2.620 \mathrm{fr}$.

Cô̂t d'implantation d'un pylône ayanl les mêmes caractérisliques, si le béton avait été employé :

10 Dressage d'un pylone de $4.500 \mathrm{kgs} . \ldots \ldots \ldots \ldots$.

$450 \mathrm{fr}$

$2^{\circ}$ Supplément de terrassement en plus du bloc de béton, qui n'est pas un cube parfait .........

$3^{\circ}$ Evacuation de $20 \mathrm{~m} 3$ de terre à $5 \mathrm{fr} . \ldots \ldots \ldots \ldots$

$4^{\circ}$ Cube de béton nécessaire pour assurer une stabilité de 2 , alors que, dans le cas précédent, elle est supérieure à $4(25 \mathrm{~m} 3$. à fr. $120 \ldots \ldots \ldots \ldots \ldots$ (Moment de renversement $60.000 \mathrm{kgr}$.)

$\frac{\text { soit } 60.000 \times 2}{2}=60 \mathrm{~T}$. de béton

Total ................

$3.700 \mathrm{fr}$,

$150 "$

$100 \%$

.3 .000

Soit une différence de $1.080 \mathrm{fr}$.

Remaryue. - Les chiffres ci-dessus se rapportent à des pylones en terrain normal, ordinaire.

En résumé: Cette liaison d'environ $100 \mathrm{~km}$. représente une 
des plus grandes lignes actuelles de transport d'énergie, comparable en tous points à celles construites en Amérique. L'exécution de tels travaux est très rapide et fait honneur au secteur qui l'a entreprise.

L'utilisation des forces du Rhin amènera bientôt la construction de lignes semblables dans l'inlérèt même de tousl es Français. Les secteurs devront inaugurer une politique nouvelle de contrats et de vente d'énergie pour la répandre à profusion, et il est à souhaiter que des groupements de secteurs se fassent, comme cela a ėté tenté en Suisse (Voir article de M.de Watteville, dans la $H . B$. de mars-avril $1922, \mathrm{n}^{\circ} 13$ ), pour jouer le rôle de chef de distribution en ajustant les puissances des différences centrales créées, aux variations de consommation dans une journée et suivant les régions.

Il est aussi à souhaiter que les capitaux engagés et les efforts faits pour construire de tels transports le soient suivant un plan général d'ensemble, dont chacun pourrait tirer le meilleur profit.

\title{
Méthode thermométrique de Mesure de Rendement des Turbines hydrauliques.
}

\section{Précautions à prendre pour l'Application correcte de cette Méthode.}

\author{
Par E. POIRSON, Ingénieu" électricien I. E. G.
}

La méthode thermométrique do mesure du rendement des lurbines, qui a fait l'objet de nombreuses publications dans les revues techniques et qui, notamment dans La Houille Blanche de septembre-octobre 1920 et de novembre-décembre 1921, a donné matière à des publications de résultats très complets, suppose, comme on le sait, l'exécution de quelques mesures très simples, au moins théoriquement, des prises de température de l'eau à la sortie des turbines, à leur entrée, et cnfin de l'eau détendue soutirée de la conduite forcée.

Cette méthode, connue déjà sous le nom de Méthode de MM. Barbillion et Poirson, ses deux auteurs, a fait l'objet de nombreuses applications dans des usines de chûtes diverses. On peut dire que, tant que la chûte reste supérieure à $40 \mathrm{~m}$. environ, la méthode s'applique avec une réelle facilité et dans des conditions lout à fait remarquables de précision.

Cependant, il importe, pour que cette précision soit effective, qu'un certain nombre de précautions soient prises, qui ne peuvent être indiquées que comme le fruit d'une longue expérience. Ayant eu personnellement à appliquer la méthode dans le plus grand nombre de cas, au cours d'expériences faites dans les usines de Sociétés dont nous avions le contrôle, nous avons cru bon de résumer ci-après l'ensemble de la doctrine opératoire à observer croyant ainsi rendre service à nos collègues expérimentateurs dans la matière. En particulier, nous citerons les expériences très intéressantes, faites aux usines de Fully (Suisse), sous la direction de M. Boucher. La chûte de Fully est actuellement la chûte la plus haute équipée dans le monde $(1.631 \mathrm{~m}$.). Les résultats trouvés par les ingénièurs de la Société exploitant la chûte de Fully ont été de tous points comparables à ceux que nous avons obtenus nous-mêmes au cours de nombreuses expériences.

La formule : $r_{1}=\frac{427\left(\Theta_{2}^{\prime}-\Theta_{2}\right)}{H}$ donne le rendement avec exactitude, sous la réserve que :

$\Theta_{2}=$ Température de l'eau sortant de la turbine dans le canal de fuite,

$\Theta_{0}^{\prime}=$ Température de l'eau. détendue dans le récipient,

$\mathrm{H}^{2}=$ Hauteur manométrique,

soient mesurées correctement, et il est utile de donner à cet égard les indications pratiques suivantes :

Les causes d'erreur principales sont : a) Variations diurnes ou autres de la température $(-)$, de l'eau arrivant à la turbine,

b) Dans la mesure de $\Theta_{y}^{\prime}$, soutirage de l'eau en un mauvais endroit de la conduite forcée, ou débit insuffisant de l'eau soutirée.

c) Dans la mesure de $\theta_{2}$, influence d'une trop grande capacité, relativement au débit, du canal de fuile en amont du point où l'on relève $(\rightarrow$,

d) En hiver, pour les températures voisines de $0^{\circ}$, influence de glaçons en suspension dans l'eau, et faussanl l'échauffement de l'eau,

e) Erreurs de mesures thermométriques et manométriques,

f) Mesures trop hâtives des températures avant la stabilisation des régimes.

Voici à cet égard les précautions élémentaires à prendre:

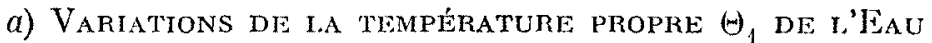

$1^{\circ}$ Variations diurnes. - Il est un fait connu dans les régions montagneuses surtout, c'est que la température des eaux suit. les variations diurnes de la tempćrature ambiante, c'est-àdire varie d'une façon continue et relativement lente. Il y a chaque jour un maximum et un minimum de température.

Les variations peuvent atteindre $1^{\circ}$. à $2^{\circ}$ par heure, soit $0^{\circ}, 017$ à 00,034 par minute, ce qui est appréciable lorsque les inesures demandent quelques minutes et portent sur des différences à mesurer de $0^{\circ}, 100$ à $1^{\circ}$, généralement.

$2^{\circ}$ Coups de soleil. - En deuxième lieu, les alternances de soleil et d'ombre causées par les nuages, sur le canal d'amenée ou les conduites forcées, se traduisent immédiatement par des variations correspondantes de la température de l'eau. Celles-ci sont plus rapides que les diurnes et il faut éviter d'opérer en pareilles circonstances où il est plus difficile de saisir les températures instantanées.

$3^{\circ}$ Arrêts. - Enfin, Jorsque une usine a été arrêtée un certain temps, l'eau en quantité importante arrêtée dans les conduites et le canal d'amenée prend la température ambiante. Lorsque on remet en marche, cette eau met un certain temps pour s'écouler, et lorsque Iui succède l'eau vive du cours d'eau, il se produit à un certain moment une rapide variation de température. Il faut éviter, là encore, d'effectuer les mesures dans ces instants là : il faut attenđ̆re le temps suffisant que le régime stable soit établi. 